\title{
Awake Craniotomy for Left Insular Low-Grade Glioma Removal on a Patient with Learning Disabilities
}

\author{
Andrej Vranic ${ }^{1}$ Blaz Koritnik ${ }^{2}$ Jasmina Markovic-Bozic ${ }^{3}$ \\ ${ }^{1}$ Department of Neurosurgery, Fondation Ophtalmologique A. de \\ Rothschild, Paris, France \\ 2 Department of Neurophysiology, University Medical Centre, \\ Ljubljana, Slovenia \\ ${ }^{3}$ Department of Anesthesiology, University Medical Centre, \\ Ljubljana, Slovenia \\ Indian J Neurosurg 2017;6:41-43. \\ Address for correspondence Andrej Vranic, MD, PhD, Department of \\ Neurosurgery, Fondation Ophtalmologique Adolphe de Rothschild, \\ 29, Rue Manin, 75019 Paris, France (e-mail: andrejvranic@yahoo.fr).
}

\begin{abstract}
Keywords

- low-grade glioma

- awake craniotomy

- learning disability
\end{abstract}

Introduction Low-grade gliomas (LGG) are slow-growing primary brain tumors in adults, with high tropism for eloquent areas. Standard approach in treatment of LGG is awake craniotomy with intraoperative cortical mapping - a method which is usually used on adult and fully cooperative patients.

Case Report We present the case of a patient with learning disabilities (PLD) who was operated for left insular LGG awake craniotomy, and intraoperative cortical mapping were performed and the tumor was gross totally removed.

Conclusion Awake surgery for left insular LGG removal is challenging; however, it can be performed safely and successfully on PLD.

\section{Introduction}

Low-grade gliomas (LGG) are slow-growing primary brain tumors in adults. For many decades, these tumors were considered inoperable because of their high tropism for eloquent areas and white matter pathways. However, patients' young age and the inescapable anaplastic transformation of LGG have recently suggested a more aggressive treatment approach, strongly arguing in favor of achieving maximal resection as the first therapeutic option. A more aggressive resection of LGG predicts significant improvement in overall survival compared with simple debulking or just biopsy. ${ }^{1,2}$ Extended removal of margins beyond the magnetic resonance imaging (MRI)-defined abnormalities (supra-total tumor resection) additionally increases overall survival by delaying anaplastic transformation. ${ }^{3}$

Introduction of pre- and intraoperative functional diagnostics of brain cortex, including awake craniotomy, has improved safety of the resection. Consequently, tumors which were previously suitable only for biopsy and follow-up are now approached with the purpose to be gross totally removed. It has been shown that awake brain tumor surgery can be safely performed with extremely low complication and failure rates regardless of American Society of Anesthesiologists classification, body mass index, smoking status, psychiatric or emotional history, seizure frequency and duration, tumor site, size, and pathology. ${ }^{4}$ However, this method is generally reserved for adult cooperative patients and no data about performing awake craniotomy on a patient with learning disabilities (PLD) is available in the literature.

In this report, we describe the case of a 39-years-old male PLD with left insular LGG. Subtotal tumor removal was performed under scalp block combined with dexmedetomidine sedation (MAC technique, monitored anesthesia care). To our best knowledge, this is the first documented case of a PLD who underwent awake brain tumor surgery.

\section{Case Report}

A 39-year-old man presented with intractable epileptic fits and a tumor in the left insular region. The patient had been treated received

March 1, 2016

accepted

April 20, 2016

published online

February 10, 2017
DOI http://dx.doi.org/

10.1055/s-0036-1585461. ISSN 2277-954X.
(C) 2016 Neurological Surgeons' Society of India
License terms

(요 (1) $\Theta \circledast$ 
for meningitis at the age of 4 years and remained intellectually impaired thereafter. His intelligence quotient (IQ) was evaluated between 60 and 70 by pediatricians. The patient was defined as a child with learning disability and he finished primary school for children with learning disabilities. Described by his parents as "a bit slow but guidable" person, he remained without epileptic fits until the age of 34 years when grand mal fits started. They were first attributed to meningitis sequels; however, after a couple of months, computed tomography and MRI were performed, revealing a large tumor in the left hemisphere. The homogenous lesion involved the left insula, as well as the left frontal and the left temporal opercular regions and was suggestive of an LGG. Follow-up was proposed and the patient was followed-up with serial yearly MRI scans during the next 4 years. Tumor growth was slow, defined as around 2 to $3 \mathrm{~mm}$ per year. At the age of 38 years, needle biopsy was performed at another institution, the histology report showing pilocytic astrocytoma. After biopsy, the patient became aggressive, with more frequent generalized epileptic fits (at least twice a week), in spite of combination therapy with three antiepileptic drugs.

Due to further growth and since the histopathological diagnosis was improbable according to the MRI images, following treatment options were considered:

- Further follow-up with yearly control MRI scans.

- Second needle biopsy, followed by radio and chemotherapy if histology would have showed grade II or III glioma.

- Maximal tumor reduction under general anesthesia.

- Maximal tumor reduction under regional anesthesia.

Considering the patient too difficult to control if operated awake, tumor reduction under general anesthesia was considered first. However, after a thorough discussion with the patient and his family, a decision was taken to operate the patient with help of awake craniotomy. Standard pre- and intraoperative protocol was used, as in other patients with LGG in eloquent regions. Throughout the surgery, sedation was maintained with dexmedetomidine, while during painful phases boluses of remifentanil were added. No use of manual restraint was necessary. Ramsay sedation scoring (target value between 2 and 3) and Bispectral Index Scale (BIS; target value more then 60) were used for sedation monitoring. Cortical mapping was performed during the critical phase, helping to determine areas of speech and motor arrest. Surgery was uneventful, and the patient remained awake, cooperative, and in good mood throughout the whole procedure. No intraoperative epileptic fits and no need for general anesthesia appeared. Tumor was successfully subtotally removed and the patient tolerated surgery well. After the procedure, he remained in the intensive care unit for 3 days. He recovered well and fast, without any additional neurological deficits or any other complications. Thereafter, he remained seizure-free, although with same antiepileptic treatment as before surgery, postoperative MRI scanning showing around $80 \%$ of the tumor removed. He was released from the hospital on 7th postoperative day. Three months after surgery, at a control check-up, the parents described slight memory deterioration, without any other neurologic deficits, and no epileptic fits.

\section{Discussion}

Ethical neurosurgical care for PLD is a hot topic. Due to significant number of PLD being subjected to lobotomy in the past, we must be particularly careful when dealing with these patients. ${ }^{5}$ In the United States, the Americans with Disabilities Act guarantees equal access to facilities, including hospital care, for PLD. However, brain surgery on awake PLD would in most cases be rejected due to presumed higher possibility of patient becoming agitated and uncontrollable during surgery.

Since awake craniotomy can safely be performed on pediatric population, ${ }^{6}$ there is no reason why this procedure could not be performed on carefully selected PLD. So, if an individual has a learning disability, but is otherwise suitable for an awake craniotomy, why not proceed? In our case, performing surgery on awake PLD turned out to be even less difficult than performing the same procedure on a patient with a normal or supra-normal IQ. Contrary to what we had expected, our patient expressed less anxiety than patients without learning disability, most probably because he felt confident and he trusted the medical team.

Each patient needs to be assessed carefully preoperatively for their emotional suitability for an awake craniotomy. Our patient was an adult patient, aware of what was going on, and even if his legal status allowed his parents to sign the consent, the patient's accordance and cooperation were mandatory to perform surgery safely. While adult PLD who are candidates for awake craniotomy are very rare, similar pediatric cases are much more frequent. In pediatric PLD, an ethical dilemma whether to operate on a patient with a complex surgical indication (as in large tumor close to eloquent areas) might lead to postponing surgery several times. During the waiting time, the patient can become a grown-up person, and being major, his legal status can change. In a suboptimal behavioral situation, he could decline surgery.

As in patients without learning difficulties, no use of manual restraint was necessary during surgery in our case. The same approach is usually used in other medical fields. In dental care of PLD, behavior management techniques and sedation are frequently used, dentists in general rejecting the restraint of PLD. However, limited use of manual restraint in accordance with parents or carers seems to be accepted, ${ }^{7}$ and occasional temporal sedation is sometimes needed.

The aim of anesthetic management is to make surgery safe and effective (airway, intracranial pressure, surgery, and mapping) and to reduce stress to the patient (preventing pain, seizures, vomiting, sedation, and comfort). Given the intrinsic stress of an awake procedure, the anesthesiologist needs to be prepared to convert to an asleep procedure. We found the MAC technique the most appropriate for our patients. Combination of scalp block with dexmedetomidine enables cooperative sedation, anxiolysis without agitation, and analgesia without respiratory depression. ${ }^{8,9}$ Constant anesthesiological monitoring as in all cases with awake 
surgery is mandatory in PLD, and vigilance for possible general anesthesia should be even more expressed. Ramsay scoring and BIS monitoring are now widely used as instrumental tools for sedation monitoring due to their reliability and applicability. BIS scores during general anesthesia were showed not to be affected by degree of learning disability, and there were no significant differences in slope of induction of anesthesia. However, the slope of emergence from anesthesia leading to tracheal extubation showed a significantly longer emergence time in more disabled patients. ${ }^{10}$ Continuous intensive postoperative monitoring of vital signs, as well as regular determination of Aldrete score, management of postoperative nausea and vomiting, pain control, and frequent neurologic examination, are all very important to avoid life-threatening complications in PLD.

As LGG per se very frequently cause epileptic fits, PLD harboring LGG are under very high risk to develop prolonged epilepsy seizure during intraoperative cortical stimulation. Cold saline must be prepared to stop seizures immediately.

Next to LGG resection, the two most common neurosurgical procedures that call for an awake patient include epilepsy surgery and functional neurosurgery. ${ }^{8}$ Epilepsy is a common comorbidity among PLD. Clusters of seizures, prolonged seizures, and status epilepticus occur more frequently in PLD. Chronic seizures, including those caused by tumors, are also more likely to be refractory to treatment. Medical treatment can interfere with cognition and cause behavioral disturbances, making life very difficult for patient and his family. In many (especially pediatric) PLD, seizures appear to further contribute to mental retardation, so special considerations apply to treatment of epilepsy in PLD. In carefully selected patients with specific epilepsy syndromes, epilepsy surgery can provide partial or complete relief from seizures. ${ }^{11}$ Monitoring patients in the awake state allows more aggressive resection of epileptogenic foci in functionally important brain regions.

No attempt for total tumor removal was attempted in our case; however, the tumor burden was diminished for $\sim 80 \%$. This is in consistency with a concept of LGG being a chronic disease, best managed in tailored approach. ${ }^{3}$ Nowadays, patients with LGG should have a normal life with a part of tumor still present, however, they have to be submitted to regular check-ups and MRI scans. Several surgical, chemotherapeutic, and radiotherapeutic procedures are usually needed, following each other over many years. Awake craniotomy is mandatory to preserve the patients' quality of life, especially in cases where tumor is situated next to eloquent regions of the brain. Help of an awake patient and continuous monitoring of brain function during surgery offers safety and enables more thorough resection. While in our case there is no technical advance, operative nuance, or unique pathology to report, we think that it is important to stress that awake surgery with tumor removal can be performed successfully in a
PLD. Although by a report of success in a single case we cannot conclude that this technique is safe for PLD, the patient tolerated the procedure well, and it proved safe enough to use the approach further. Ongoing experience will bring additional improvement in the protocol and possibly expand indications for using awake craniotomy.

\section{Conclusion}

Awake craniotomy can be performed safely and successfully on patients with learning disability.

\section{Conflict of Interest}

The authors state they have no conflicts of interests.

\section{References}

1 Capelle L, Fontaine D, Mandonnet E, et al; French Réseau d'Étude des Gliomes. Spontaneous and therapeutic prognostic factors in adult hemispheric World Health Organization Grade II gliomas: a series of 1097 cases: clinical article. J Neurosurg 2013;118(6): 1157-1168

2 Jakola AS, Myrmel KS, Kloster R, et al. Comparison of a strategy favoring early surgical resection vs a strategy favoring watchful waiting in low-grade gliomas. JAMA 2012;308(18):1881-1888

3 Duffau H. A new philosophy in surgery for diffuse low-grade glioma (DLGG): oncological and functional outcomes. Neurochirurgie 2013;59(1):2-8

4 Hervey-Jumper SL, Li J, Lau D, et al. Awake craniotomy to maximize glioma resection: methods and technical nuances over a 27-year period. J Neurosurg 2015;123(2):325-339

5 Ogren K, Sjöström S, Bengtsson NO. The unknown history of lobotomy: women, children and idiots were lobotomized [in Swedish]. Lakartidningen 2000;97(30-31):3395-3398

6 Balogun JA, Khan OH, Taylor M, et al. Pediatric awake craniotomy and intra-operative stimulation mapping. J Clin Neurosci 2014; 21(11):1891-1894

7 Marks L, Adler N, Blom-Reukers H, Elhorst JH, KraaijenhagenOostinga A, Vanobbergen J. Ethics on the dental treatment of patients with mental disability: results of a Netherlands Belgium survey. J Forensic Odontostomatol 2012;30 (Suppl 1):21-28

8 Erickson KM, Cole DJ. Anesthetic considerations for awake craniotomy for epilepsy and functional neurosurgery. Anesthesiol Clin 2012;30(2):241-268

9 Conte V, Baratta P, Tomaselli P, Songa V, Magni L, Stocchetti N. Awake neurosurgery: an update. Minerva Anestesiol 2008;74(6): 289-292

10 Ponnudurai RN, Clarke-Moore A, Ekulide I, et al. A prospective study of bispectral index scoring in mentally retarded patients receiving general anesthesia. J Clin Anesth 2010;22(6): 432-436

11 Shields WD. Management of epilepsy in mentally retarded children using the newer antiepileptic drugs, vagus nerve stimulation, and surgery. J Child Neurol 2004;19(Suppl 1): S58-S64 